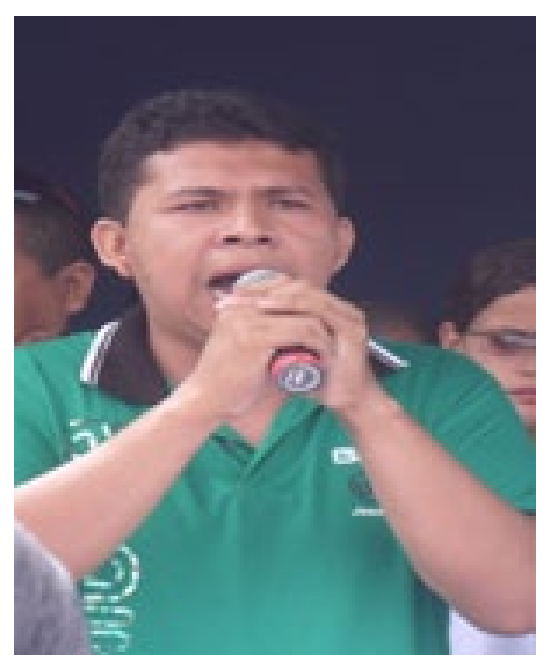

Fotografia 16: Gildiere Ribeiro Pereira - Pajé da etnia Fulni-ô e professor de Língua Materna - Yaathê

\section{APRENDIZAJE \\ Y COMPETENCIAS \\ INTERCULTURALES}

Alessio Surian alessio.surian@unipd.it

\section{Resumen}

El concepto de competencia intercultural es asociado en la literatura tanto a listas de habilidades que permiten tener "éxito" como individuo y como organización en las relaciones transculturales, como visiones más criticas e interesadas a los diferentes contextos y papeles sociales que juegan un rol en la definición y percepción de estas relaciones.

Al concepto de competencia intercultural autores como Earley y Ang (2003, p. 59) prefieren el concepto de inteligencia cultural, en relación a como las personas saben adaptarse a nuevos contextos culturales, y en continuidad con el trabajo sobre inteligencias de psicólogos de la educación (H. Gardner, R.J. Sternberg), y en relación a la interacción entre estilos cognitivos y gestión de las actividades cotidianas (Zhang, Sternberg, 2001, pp. 198-200).

A partir de enfoques diferentes, se ha producido en los últimos años un numero significativo de herramientas de evaluación de las competencias interculturales (Earley y Ang 2003, pp. 193-199; Fowler y Blohm 2004, pp. 37-84; Paige 2004, pp. 85-128).

En esta contribución se investigan los aspectos relevantes y las implicaciones de los discursos sobre competencias interculturales para los procesos de aprendizaje y para las políticas de educación que integran un enfoque intercultural.

Palabras claves: relaciones transculturales; políticas de educación Key words: crosscultural relations; educational policies 
Amaras otra vez el extranjero que ha sido tu Yo Derek Walkott ("Amor después Amor")

\section{INTRODUCCIÓN}

$E^{d e}$ ste ensayo ententa mapear las dimensiones relevantes en terminos de apredizaje en relación a la "interculturalidad" sin ententar darle la expresión "interculturalidad" un sentido univoco. Escrito a partir de la experiencia del autor con los estudios academicos y los programas de formación interculturales, este mapeamento pone en evidencia algunos de los concpetos claves en Europa para facilitar un intercambio con otros contextos de aprendizaje reconociendo con Mato (2008) y Sandoval (2011: 3) las luchas y los logros en las Americas de "los indígenas (...) por un modelo educativo acorde a su realidad, a sus condiciones y saberes heredados. Una educación intercultural ejercida desde y para los indígenas, aquella que mediante la decolonizaciónización y la enseñanza fundada en la cultura de cada grupo contribuya a la formación de sujetos interculturales para la paz, lo cual obliga a romper con el modelo occidental en la enseñanza para dar cabida a otras formas de generar conocimiento y modos de emplearlo en la solución de problemas cotidianos".

En el ámbito de la "educación intercultural bilingüe" la denominación "interculturalidad" asume un sentido positivo. "No obstante, debe decirse que en principio se trata de una categoría meramente descriptiva que refiere de manera genérica al campo de las 'relaciones entre culturalmente diferentes'. Estas relaciones pueden asumir diversos signos. Las sociedades contemporáneas son 'interculturales de hecho'. Esa 'interculturalidad de hecho' frecuentemente reviste contornos sumamente negativos, que desafortunadamente incluyen formas de racismo y desvalorización de diferencias culturales significativas" (Mato 2008: 15)

En general, entre las primeras sugerencias para quienes enfrentan contextos culturales que puedan proporcionar dificultades encontramos la idea de que es útil salir de formas de pensamiento demasiado lineal; también nos ayuda aprender a analizar el contexto que acoge comunicación y conductas, tal como preguntarse sobre el posible sentido en relación a la comunicación de aspectos que inicialmente aparezcan como marginales.

¿Cuales son las huellas significativas y como se conectan entre si? A la idea de huella y de trayectoria le han dedicado análisis significativos antropólog*s que dan atención a las condiciones ecológicas de nuestras acciones culturales. Entre ell*s, Tim Ingold (2006) sugiere que hay varias maneras de mirar a lo que consideramos "línea": posiciones, gestos y trayectorias que parecen lineales siempre merecen ser analizados como si no lo fueran. Ingold nos habla de las transformaciones de la manera de viajar, mapear, contar, que marcan la modernidad como el tiempo en que la tendencia dominante es percibir las personas como entidades que "ocupan" un espacio, pero siempre queda útil caminar y parar a través de los espacios para recuperar la percepción de quienes nos rodean como moradores de su propio ambiente, encontrar líneasconexiones y no simplemente líneas-segmentos que identifican posiciones. ¿De que manera el concepto de línea afecta también las siguientes paginas? Para responder, intentamos pensar primero la idea de objetivo: muchas veces esto es lo mas importante para medir competencias y su eficacia.

Fijarse en un objetivo. Es una acción generalmente considerada útil y necesaria. Al mismo tiempo, ya su formulación, tomar conciencia para un organismo de la necesidad de concentrar atención y esfuerzos hacia un preciso punto focal, nos señala una dificultad para perseguir esta tarea. Sobre todo cuando un objetivo final tiene que ser dividido en objetivos intermedios, la acción de fijarse en un objetivo implica un riesgo potencial. Eso lo explica de manera clara el escritor Deon Meyer (2004: 122) analizando los comportamientos de quienes viajan en moto: "El mayor enemigo de quien viaja en motocicleta es fijarse en un objetivo. Es algo instintivo. Desafortunadamente el enlace entre ojos y cerebro funciona de esta manera: si miras un hueco o una piedra terminaras en encontrarla. No tienes que mirar nunca el obstáculo directamente. (...) Cuando hayas identificado un obstáculo en la carretera, tienes conciencia que existe y que hay que buscar una manera de pasarlo alrededor; entonces hay que fijar los ojos en una línea de seguridad y automáticamente tu y tu moto la van a perseguir".

En relación a la necesidad de preguntarnos sobre los significados que le damos a las competencias interculturales, esta orientación a una ampliación perceptiva 
pide un análisis de la comunicación y de los comportamientos que incluya perspectiva ex quo tal como la perspectiva ad quem explicadas por filósofos como Severino (2007: 107): esto para resistir a concepciones del significado mas limitadas o dicotómicas, por ejemplo a la contraposición sugeridas por los académicos de la comunicación como Frege (1978: 9-32), entre sentido y significado.

Las siguientes paginas intentan adoptar esta orientación a una línea de seguridad, el intento de mirar mas allá como metáfora de la competencia intercultural y de la capacidad de saber reconocer "indicios de contextualización" (Chick, 1990) y para saberse mover entre los marcos que identifican tratos culturales.

\section{Territorios de frontera, piedras y pequeñas piedras}

¿Que metodologías son hoy las más populares entre los profesionales de las consultorías y de la formación intercultural? En un estudio realizado para SIETAR Europa por Berardo e Simons (2004: 14-15) se encuentran sobre todo modelos descriptivos, estudio de casos, ejercicios, juegos de simulación. Entonces, se trata sobre todo de abordar la materia a partir de la experiencia, en particular de los participantes, dándole atención a los incidentes críticos y a las reflexiones de las ciencias sociales sobre los territorios de frontera.

En las ciencias sociales la reflexión sobre los dispositivos que permiten y facilitan moverse a través de las cornisas culturales esta viviendo una fase de aceleración en relación a los procesos de territorialización que afectan los flujos culturales, informacionales, simbólicos y, al mismo tiempo, económicos (Appadurai, 1996). Los territorios de frontera ofrecen oportunidades para detectar lo que es inadecuado en las concepciones culturales que entran en contacto y en un proceso de comparación.

En general observamos rapidez y claridad en la manera que tiene una persona de identificar los limites de los mundos que le resultan menos familiares pero la misma persona suele necesitar de mucho mas tiempo para enfrentarse y enterarse con sus propios conceptos del mundo (Mantovani, 1998: 144). Es la distancia entre la capacidad de adscripción y de inscripción (Pierik, 2004 532). En la sistematización de algunas investigaciones sobre comunicación intercultural y aspectos ideológicos en los servicios sanitarios y sociales, Van
Asperen (2003: 118-119) nos habla del juicio expresado por una trabajadora social holandesa que es un ejemplo de estas dos acciones, empezando por la segunda: "En una familia holandesa se ve como natural que los niños ayuden en los trabajos de la casa (...). En las familias de Marruecos esto no es así cuando se trata de un niño porqué los niños de sexo masculino no ayudan en los trabajos de la casa". La diferente capacidad de interpretación de las categorías culturales próximas o lejanas nos hace pensar de que hablamos cuando hablamos de "competencia", un concepto "multiprospectivo" (Viteritti, 2005): un saber en acción que nos hace concientes de como la acción trasforma los contextos sociales. Cada persona no se relaciona con los contextos sociales con libertad absoluta: cada persona ve en los contextos sociales "reglas" que responden a sus propias ideas culturales de base (Mantovani, 1995:. 91; Sahlins, 1985: ix).

En esta perspectiva, la capacidad de actuar en relación a (Newell, Simon, 1972) y de comprender un determinado marco cultural es en relación con reglas "locales" de tipo general, y con reglas "especificas" del contexto comunicativo. El escritor Kader Abdolah (2007: 35) nos cuenta hablando de si mismo y de la forma de expresarse de su gente: "Los persas hablan de forma vaga, velada. Nuestra cultura nos no permite expresar completamente nuestro pensamiento". Valsiner (2000: 46) afirma que los procesos de "tribalización" afectan también los estudios culturales y que hoy se han formados grupos de vygotskyianos, de bachtinianos, de meadianos y de simmelianos y pone en relación esto a lo que para los antropólogos es el "cargo cult", la idea que bienes importados desde lugares prestigiosos puedan desvelar cualidades mágicas para solucionar los problemas de los afiliados a un culto especifico. En perspectiva dinámica, la reflexión filosófica de Ricoeur (1965) nos sugiere un análisis de los discursos identitarios como secuencias de "rectificaciones y correcciones de una serie de cuentos", y no como algo hecho "cosa": cada cuento es "síntesis del heterogéneo".

El conocimiento y la consciencia socio-cultural de los marcos de los discursos (Bateson, 1955) nos hablan de la capacidad de des-aprendizaje, a los diferentes niveles en que se traduce la inserción en un contexto (Cole, 1989) y a la metáfora del juego, en la medida que "cada juego nos pide aceptar por un tiempo (...) un universo cerrado, convencional (...) la persona juega a creer, a ser creído o a hacer creer a los demás de ser otra persona. La persona niega, muda, abandona por un tiempo su propia personalidad para hacer muestra 
de ser otra. (...) He elegido citar estas manifestaciones con la palabra mimicry, palabra inglesa que indica el mimetismo, en particular de los insectos, para subrayar la naturaleza fundamental y elementar, cuasi orgánica, del impulso que genera" (Callois, 1967).

\section{Sobre el concepto de competencia}

Como practica social y saber en acción, las ciencias sociales ven las competencias como espacio de interpretación donde, a nivel individual y colectivo, se expresan experiencia, cognición y voluntad (Viteritti, 2005). La literatura nos ofrece una progresiva ampliación del concepto de "competencia intercultural" (Deardorff, 2004, 2009). Este espacio de investigación se ha desarrollado sobre todo en Norte América a partir de los años cincuenta del siglo pasado, dándole atención a las dinamicas del shock cultural (Oberg, 1960) y a dimensiones consideradas menos explicitas en la comunicación interpersonal tal como la comunicación nonverbal y la proxemica (E.T. Hall, 1959, 1966).

En las últimas décadas, la reflexión sobre comunicación y competencia intercultural no se ha limitado al repertorio de acciones a disposición de las personas que se encuentran en contextos culturales disueltos, sino ha analizado también sus especificas representaciones de la realidad y de las relaciones sociales. Por ejemplo, el sociólogo estadunidense Milton Bennett nos habla de mindset, las maneras de mirar el mundo (las actitudes), y de skillset, las praticas y las competencias que se necesitan para la acción. Esta perspectiva puede ser desarrollada tomando en consideración la "reciproca implicación" entre los conceptos chinos de "hun" y "po": "hun nos habla de lo que es alto por inteligencia, conocimiento, sensibilidad, espiritualidad, reflexión, creatividad, y contemplación; po nos habla de todo lo que es 'zavora' para estas actividades, que las enracima entre limites específicos y ámbitos determinados, sin que se difuminen, se apaguen o resulten ineficaces" (Pasqualotto, 2007: 124).

En un estudio sobre las actividades de educación, la investigadora estadunidense Deardorff (2004: 122) identifica más de diez conceptos sinónimos de "competencia intercultural" (cross-cultural adaptation, intercultural sensitivity, multicultural competence, transcultural competence, cross-cultural effectiveness, international competence, global literacy, global citizenship, cultural competence, y crosscultural adjustment). Los mas frecuentemente utilizados son cross-cultural o global competence, pero el concepto de "competencia intercultural" sigue siendo el mas apropiado en este ámbito (Kim, 2001).

Los investigadores y consultores Earley e Ang (2003: 264) agrupan las principales competencias interculturales en diez categorías: habilidades comunicativas; tolerancia de la ambigüedad; empatía; abertura mental; flexibilidad; capacidad de saber enfocar una tarea o una relación; postura positiva hacia el aprendizaje; tolerancia hacían estilos y culturas diferentes; conocimiento cultural; capacidad de tener éxito en contextos diferentes. Un trabajo similar ya se encontraba en literatura con categorías referidas a objetivos, habilidades comunicativas, habilidades relacionales Spitzberg y Cupach (1984: 70-71).

\section{Dimensiones de la competencia intercultural}

Los sociólogos Berger y Luckmann (1967,: 71) ponen en evidencia como los mecanismos de hábito podrían ser analizados como formas de pre-definición y previsión: en primer lugar permiten minimizar la inversión de tiempo y energía en relación a la toma de decisión, puesto que se puede evitar de definir cada situación y sus componentes específicos. Algo central en la competencia intercultural es la necesidad de discutir los estereotipos ya adquiridos (de quienes somos poco o nada conscientes): la atención del mundo de la investigación científica por este tema es algo relativamente reciente limitado sobre todo a una perspectiva dirigida al rendimiento individual y a las bases mentales de dicho rendimiento

El International Journal of Intercultural Relations le ha dedicado toda una edición de la revista al tema de las competencias interculturales (Martin, 1989). Las contribuciones hablan de un modelo articulado en seis dimensiones principales, que sirven todavía de base a la literatura sobre la formación de la experiencia intercultural (Paige, 2003b: 171). Según este modelo, la "eficacia intercultural" es influenciada de manera positiva por seis grupos de factores: elementos en relación al conocimiento de la cultura "target"; cualidades personales tal como abertura, flexibilidad, tolerancia de la ambigüedad y humor; habilidades comportamentales tal como la eficacia en la comunicación, actitudes culturalmente adecuadas a los roles y posturas relacionales; consciencia de si, y específicamente de valores y convicciones; habilidades 
técnicas como la capacidad de llevar a cabo una tarea en el nuevo contexto cultural; factores relativos al nuevo contexto tal como la semejanza relativa con la cultura de origen, la forma de acoger el extranjero, las condiciones socioeconómicas y políticas de la "segunda" cultura, el nivel de claridad en relación a las expectativas en relación al rol y a la posición del extranjero, la presión psicológica asociada a la experiencia.

Se trata de un modelo que no desarrolla un pensamiento sobre las competencias interculturales sino trabaja a nivel de descripción de su marco operacional con enfoque dicotómico: sujeto / nuevo contexto local. Típico de este modelo es el uso de términos como "technical skills" (habilidades tecnicas) y "knowledge of target culture" (conocimiento de una determinada cultura): se trata de un abordaje culture-specific, orientado a ser eficaz en un determinado contexto cultural.

Un abordaje sistemático del tema es presentado por la investigadora estadunidense $\operatorname{Kim}(1992,2001)$, que trata la competencia intercultural como capacidad de adaptación en ámbito cognitivo (dar sentido), afectivo ("tendencias" emotivas y estéticas, predisposiciones motivacionales y actitudinales), y operacional/comportamental (nivel de flexibilidad y recursos a disposición). Kim habla de capacidad de adaptación como "capacidad del individuo de suspender o mudar algunas modalidades culturales adquiridas y de aprender y acomodar algunas de las nuevas modalidad culturales, encontrando creativamente maneras de gestionar las dinámicas relativas a los papeles inter-grupo diferentes y el estrés asociado" (Kim, 1992:. 377). Como Spitzberg y Cupach (1984: 90) Kim (1992: 373), considera la capacidad de adaptar la capacidad de adaptación como comportamiento y habilidad que el individuo sabe practicar en contextos diferentes y en este sentido hay una diferencia significativa entre la competencia cultural culture-specific y la competencia intercultural que suele resultar eficaz de manera independiente de los específicos contextos culturales.

La articulación de la competencia intercultural en conocimientos, habilidad y actitudes domina la mayoría de las definiciones. Imahori e Lanigan (1989: 276277) presentan una interesante excepción porque por ellos la "competencia intercultural es en relación a un "adecuado nivel de motivación, conocimiento $y$ habilidades de quien se encuentra en otro contexto y de las personas de este nuevo contexto en relación a los intercambios que establecen y que llevan a resultados relacionales eficaces". En este modelo de competencia intercultural ambos actores entran en contacto y hay interacción en términos de nivel de motivación, conocimiento y habilidades y ambos están influenciados por objetivos y por vivencias pasadas que resultan específicas por cada persona. En este modelo se evidencian los aspectos de interacción de la acción transcultural. No se trata de un modelo comúnmente aceptado, si pensamos que el proyecto Intercultural Competence Assessment (INCA) financiado por el Programa Leonardo de la Comisión Europea (Byram, 1997) limita el concepto de competencia intercultural a la capacidad de interacción eficaz y aceptable por parte de los otros, poniendo la atención sobre capacidades lingüísticas, identidad y comprensión cultural.

Dos ejemplos de modelos evolutivos de las competencias interculturales se encuentran en el trabajo de Hoopes (1979) y Milton Bennett (1993). Estos modelos trabajan un continuum que empieza con la condición etnocentrismo y desarrolla a través de algunas etapas una plena consciencia y competencia intercultural. El modelo de Bennett (1993) llamado Developmental Model of Intercultural Sensitivity (DMIS) identifica tres etapas etnocéntricas rechazo, defensa, minimización) y tres etapas etnorelativas (aceptación, adaptación, integración). Según Bennett (1993: 46), la postura etnorelativa es una posición que considera que "las culturas solo se puedan entender en relación entre ellas y que una postura especifica solo se puede entender en su contexto cultural" (ib. : 46).

El aspecto llave de la definición "procesual" de etnorelativismo es el concepto de "empatía" (Håkansson, J., Montgomery, H., 2002). Para Bennett (1993:

53) esta es la capacidad de reconocer y apropiarse de marcos de referencia cultural diferentes de los marcos personales, "la habilidad de hacer experiencia de aspectos de la realidad de forma diferente de como se haría en la cultura personal".

Este breve resumen pone en evidencia algo subrayado también por Lustig y Koester (2003: 65): "no se puede hablar de un conjunto prescriptivo de características que puedan garantizar con certeza competencia en cualquier relación y situación intercultural". El discurso dominante sobre competencia intercultural se preocupa en primer lugar por la eficacia del individuo en 
nuevos contextos culturales, no toma en cuenta todavía en perspectivas espacio-temporal más extendidas, ni aspectos de la relación que impliquen una cultura "dominante" o las dificultades especificas en relación a los fenómenos migratorios. En este sentido se necesita ampliar la reflexión sobre competencias interculturales a partir de una perspectiva ecológico-cultural, ésta ha sido ya fundada por antropólogos como Ogbu (1981) a partir de los trabajos de Julian Steward, enfocando como se transmite socialmente y cuál es la interdependencia con el medio ambiente de las formas de comportamiento habitudinal. En esta perspectiva, Dussel (2003: 50) nos explica como solo la negación del "mito de la civilización y de la inocencia de la violencia moderna" pueda hacer posible reconocer las características de injusticia de la "praxis de sacrificio" en ámbito extra-europeo y al interior de la misma Europa, estableciendo de esta manera las bases cognitivas que nos permitan superar los límites de la "razón de emancipación".

En esto contexto, Boaventura de Sousa Santos (2001: 270-271) subraya que las prácticas sociales que no están en relación con una racionalidad científica no son necesariamente practicas "ignorantes", sino practicas de saberes rivales. En perspectiva transcultural el aspecto problemático de la racionalidad científica es que, cual saber especializado y institucionalizado, tiene la tendencia y el poder de definir situaciones y contextos de maneras no solamente descriptivas, sino también prescriptivas. En este sentido, los discursos dominantes de la ciencia moderna pueden hacer que las alternativas resulten "ausentes" o "invisibles" a partir de diferencias de escala, de tipo de perspectiva, de nivel de resolución y de ámbito temporal utilizado. Para abrir una perspectiva que sepa tomar en cuenta "ceguera" y "ausencias", Santos (2001: 271) nos propone el concepto de "doble ruptura epistemológica": no es suficiente una simple discontinuidad epistemológica que haga la distinción entre ciencia y sentido común: necesitamos también la capacidad de trasformar el saber científico en un nuevo sentido común y aprender a de-construir y trasformar el sentido común dominante y conservador en perspectiva emancipatoria. Esta perspectiva nos lleva a la necesidad de un conocimiento "prudente", que sepa identificar y superar las diferentes éticas de ser "convencidos" y entonces también las hybris generadas por "certidumbres", favoreciendo una ética de la "responsabilidad" (Artosi, Brighenti, 1998: 3).

El mismo concepto y la mayoría de las definiciones de competencia intercultural indican una marcada perspectiva occidental (Deardorff, 2004: 51; Quassoli, 2006: 66). Es claro que no se encuentran definiciones de competencia intercultural "neutrales", sin bias cultural (van Asperen, 2003: 129). Todavía, hay carencia de estudios y textos que definan la competencia intercultural desde perspectivas non occidentales. Un ejemplo de comunicación definida en el contexto de un marco cultural especifico es Yum (1994) que identifica en el contexto de Korea cinco características de la comunicación, evidentemente aquí mas en relación a la idea de grupo: empatía, sensibilidad, mensajes indirectos, ser reservados y transcendencia.

En el contexto de las investigaciones interculturales, el abordaje ético de Santos y de Artosi y Brighenti sugiere una reflexión en términos de competencia intercultural sobre como una sociedad reconoce y trata el capital cultural de los recién llegados, un tema ausente en las definiciones actuales que piensan la competencia en primer lugar como instrumento funcional a la afirmación personal del individuo. Estos tipo de definiciones denotan un modelo de sociedad de co-existencia o co-adaptación, y entonces modelos multiculturales o trans-culturales, pero no intercultural, todavía no centrados en la "coevolución" (Peter Raven y Paul Ehrlich, 2000: 78).

En este sentido es clave para las profesiones con tareas institucionales en los contextos de salud, educación, servicio social y administrativo reflexionar sobre los criterios y indicadores de competencia y sobre los modelos de referencia. Un ejemplo de perspectiva más dinámica es ofrecido por la lingüista estadunidense Ting-Toomey (2004: 238-239) al identificar tres criterios principales para averiguar competencia intercultural en el contacto directa entre dos personas: apropiación, eficacia y adaptabilidad.

El tercero criterio, la adaptabilidad pone en evidencia los comportamientos verbales y no verbales a partir de una adecuada flexibilidad cognitiva, afectiva y comportamental que sepa generar actitudes trasformativas en las situaciones interpersonales de conflicto.

Es interesante notar que al concepto de competencia intercultural Earley e Ang (2003: 59) prefieren el concepto de inteligencia cultural, la capacidad de una persona de adaptarse de forma eficaz en nuevos contextos culturales. Esta perspectiva es más cercana de los trabajos de psicólogos de la educación como 
Howard Gardner y Robert J. Sternberg (Zhang, Sternberg, 2001: 198-200). En esta perspectiva las características de eficacia en situaciones de potencial aprendizaje tienen una relación con la capacidad de adaptación a los nuevos contextos y a la solución creativa de los problemas estableciendo prioridades y pensamiento critico sobre las normas. Este modelo referencia nos permite pensar la inteligencia intercultural sin vincularla a "ventajas" en áreas especificas como, por ejemplo, las competencias lingüísticas. En este sentido hay afinidad con la idea de "inteligencia ecológica" definida en ámbito educativo por Ginger y Hendricks-Lee (1999: 73) y con la afirmación de Paulo Freire (1996: 43-44) que "Na formação permanente dos professores, o momento fundamental é o da reflexão crítica sobre a prática. É pensando críticamente a prática de hoje ou de ontem que se pode melhorar a próxima prática"

En esta perspectiva inter-transcultural (Padilha, 2004) el concepto de transformación sugeridos por autores como Ting-Toomey (2004) parece el más adecuado en los contextos educativos de la catástrofe como cambio no destructivo, como propuso René Thom que nos habla de procesos con estabilidad estructural dentro de perturbaciones que llegan a mudar un proceso/sistema: fenómenos que conciernen de cerca la idea de contacto intercultural y que parecen mejor descritos por curvas, "tendencia general".

Esta atención para la tendencia y no simplemente para expresiones numéricas de los resultados, nos lleva a pensar también en el contexto de la competencias interculturales el trabajo del etnopsiquiatra George Devereux y del antropólogo François Laplantine cuando nos ayudan a utilizar las metáforas "bastón rígido o flojo" del físico Niels Bohr para pensar la interacción entre sujeto y observador: un bastón (Bateson, 1970: 477; Mantovani, 1998: 121) llevado de manera rígida es como la extensión de la propia mano y la línea de demarcación entre observador y sujeto se encuentra en la punta lejana del observador; llevado de manera lasca es menos parte del observador y entonces coloca, perceptivamente, la línea de demarcación en la punta más próxima al mismo observador. Devereux pone en relación la reflexión de Bohr con la distinción que nos propuso el psicólogo William James sobre los conocimientos. James trata las experiencias con modalidad "bastón rígido" como knowledge about, conocimiento sobre algo, mientras trata como experiencias de acquaintance with, familiaridad con, el abordaje "flojo". Para el antropólogo Laplantine (1999: 21) el bastón rígido "nos da la ilusión de una coherencia identitaria, la idea de comprender algo 'verdadero'”.

La perspectiva intercultural puede aprovecharse de un pensamiento constructivista y multidimensional que sepa mirar a las competencias interculturales como habitus socialmente situado, un concepto ya trabajado en Francia por el antropólogo Marcel Mauss y el sociólogo Pierre Bourdieu, que subrayan la fertilidad del abordaje "flojo", rizomatico en el pensar y practicar las competencias necesarias a las relaciones trans-culturales. Este abordaje rizomatico nos sugiere una idea epistemológicamente "prudente" de cultura. Como nos dice Glissant (2007: 26), los textos que "fundan comunidades", el Antiguo Testigo, las sagas, las epopeyas africanas, son "libros de exilio y muchas veces de erranza", deseo de "salir de la raíz", indicación que apoya la critica hecha por filósofos como Deleuze y Guattari al concepto de raíz-cepo a favor del concepto de raíz-rizoma. Glissant (2007: 23) nos habla de rizoma como "raíz desmultiplicada que se extiende en redes en la tierra y en el aire, sin encontrar otro irremediable predador. En esta noción de rizoma queda el aspecto del enraizamiento, rechazando la idea de una raíz totalitaria (...) cada identidad se extiende en la relación con el Otro".

\section{Bibliografia}

- ABDOLAH K. (2003), Portretten en een oude droom, De Geus, Breda

- Appadural A. (1996), Modernity at large. Cultural dimensions of globalization, University of Minnesota Press, Minneapolis.

- ARTOSI A., BRIGHENTI A. (1998), Paradigma e mutamento. La molteplicità della transizione storica contemporanea, http://www.ces.uc.pt/bss/ documentos/Artosi-Brighenti_new.pdf.

- BATESON G. (1955), A Theory of play and fantasy, in G. BATESON (1972), Steps to an ecology of mind, Chandler, San Francisco: 177-193

- BATESON G. (1970), Form, substance and difference, in G. BATESON (1972), Steps to an ecology of mind, Ballantine Books, New York: 448-464

- BENNETT M.J. (a cura di) (2002), Principi di comunicazione interculturale, Franco Angeli, Milano.

- BERARDO K., SIMONS G. (2004), The Intercultural Profession: its profile, practices \& challenges, SIETAR Europa, (http://www.sietar-europa.org/ about_us/ICP_Survey_Report.pdf)

- BERGER, P., LUCKMANN, T. (1967), The Social Construction of Reality, 
Penguin Books, Harmondsworth.

- BYRAM, M. (1997), Teaching and assessing intercultural communicative competence, Multilingual Matters, Clavedon.

- CAILlOIS R. (1967), Les jeux et les hommes. Le masque et le vertige, Gallimard, Paris

- CASTIGLIONI I. (2005), La comunicazione interculturale: Competenze e pratiche, Carocci, Roma.

- CHICK J.K. (1990), Reflections on language, interaction, and context: micro and macro issues, in D. CARBAUGH (ed.), Cultural communication and intercultural contact (pp. 253-257), Lawrence Erlbaum, Hillsdale, NJ.

- COLE M. (1989), Cultural psychology: A once and future discipline? In J. BERMAN (ed.), Nebraska symposium on motivation: Cross cultural perspective, University of Nebraska Press, Lincoln: 279-335

- COLLIER M.J. (1989), Cultural and intercultural communication competence: current approaches and directions for future research, in "International Journal of Intercultural Relations", 13: 287-302.

- DEARDORFF D.K.B. (2004), The identification and assessment of intercultural competence as a student outcome of internationalization at institutions of higher education in the United States, PhD Thesis, Raleigh, NC.

- DEARDORFF D.K.B. (ed.) (2009), The SAGE handbook of intercultural competence, SAGE, Los Angeles

- DEVEREUX G. (1984) Dall'angoscia al metodo nelle scienze del comportamento, Severi-Treccani, Roma.

- DUSSEL E. (2003), Europa, modernidad y eurocentrismo, in Lander (comp.), La colonialidad del saber: Eurocentrismo y ciencias sociales. Perspectivas latinoamericanas (41-53), CLACSO, UNESCO, Buenos Aires.

- EARLEY P.C., ANG S. (2003), Cultural intelligence. Individual interactions across cultures, Stanford University Press, Stanford, CA.

- EHRLICH P. (2000), Human natures. Genes, cultures, and the human prospect, Washington D.C., Island Press.

- FOWLER S., BLOHM J.M. (2004), An analysis of methods for intercultural training, in D. LANDIS, J.M. BENNETT, M.J. BENNETT (eds.), Handbook of intercultural training. Third Edition (37-84), Sage, Thousand Oaks, CA.

- FREGE G. (1978), La struttura logica del linguaggio, Bompiani, Milano.

- FREIRE P. (1996) Pedagogía da autonomia, saberes necesarios à prática educativa, Paz e Terra, Sao Paulo,

- GLISSANT E. (1990), Poétique de la relation. Poétique III, Gallimard, Parig
- GUDYKUNST W.B., HAMMER M.R. (1983), Basic training design: Approaches to intercultural training, in D. LANDIS, R.W. Brislin (eds.), Handbook of intercultural training. First Edition, Pergamon, Elmsford, NY.: 118-154

- GUDYKUNST W.B., MOODY B. (eds.) (2002), Handbook of international intercultural communication, Sage, Thousand Oaks, CA.

- HÅKANSSON J., MONTGOMERY H. (2002), The role of action in empathy from the perspectives of the empathizer and the target, in "Current Research in Social Psychology", 8, 50-62.

- HALL E.T. (1959), The silent language, Doubleday, New York.

- HALL E.T. (1966), The hidden dimension, Doubleday, New York.

- HOFFMAN C., LAU I., JOHNSON D.R. (1986), The linguistic relativity of person recognition: An English-Chinese comparison, in "Journal of Personality and Social Psychology", 51, 1097-1105.

- HOOPES D. S. (1979), Intercultural communication concepts and the psychology of intercultural experience, in $\mathrm{M}$. PUSCH (ed.) Multicultural education: A cross-cultural training approach (9-38), Intercultural Press, Yarmouth, ME.

- IMAHORI T.T., LANIGAN M.J. (1989), Relational model of intercultural communication competence, in "International Journal of Intercultural Relations", 13, 269-286.

- INGOLD T. (2006) Up, across and along, in "Studies in Environmental Aesthetics and Semiotics", 51, 21-36.

- KIM Y.Y. (1992), Intercultural communication competence: A systemsthinking view, in W.B. GUDYKUNST, Y.Y. KIM (eds.), Readings on communicating with strangers: An approach to intercultural communication (371-381), McGraw-Hill, New York.

- KIM Y.Y. (2001), Becoming intercultural: An integrative theory of communication and cross-cultural adaptation, Sage, Thousand Oaks, CA.

- LANDER E. (comp.) (2003), La colonialidad del saber: eurocentrismo y ciencias sociales. Perspectivas latinoamericanas, CLACSO, UNESCO, Buenos Aires.

- $\quad$ LANDIS D., BRISLIN R.W. (eds.) (1983), Handbook of intercultural training. First Edition, Pergamon, Elmsford, NY.

- LANDIS D., BENNETT J.M., BENNETT M.J. (eds.) (2004), Handbook of intercultural training. Third Edition, Sage, Thousand Oaks, CA

- LAPLANTINE F. (1999), Je, nous et les autres, être humain au-delà des appartenances, Le Pommier. 
- LUSTIG M.W., KOESTER J. (2003), Intercultural competence: Interpersonal communication across cultures (4a ed.), Allyn \& Bacon, Boston.

- MANTOVANI G. (1995), Comunicazione e identità. Dalle situazion quotidiane agli ambienti virtuali, II Mulino, Bologna.

- MANTOVANI G. (1998), L'elefante invisibile. Tra negazione e affermazione delle diversità: Scontri e incontri multiculturali, Giunti, Firenze.

- MARTIN J.N. (ed.) (1989) Intercultural communication competence, in "International Journal of Intercultural Relations", 13, 3.

- MATO D. (ed.) (2008) Diversidad Cultural e Interculturalidad en Educación Superior. Experiencias en América Latina, IESALC-UNESCO, Caracas.

- MEYER D. (2004) Infanta, Feniks, Capetown.

- NEWELL A., SIMON H.A. (1972), Human problem solving, Prentice-Hall, Englewood Cliffs

- OBERG K. (1960), Cultural shock: Adjustment to new cultural environments, in "Practical Anthropology", 7, 177-182.

- OGBU J.U. (1981), Origins of human competence, in "Child Development", 52, pp. 413-429.

- PADILHA P.R. (2004), Currículo e Educação Intertranscultural, IPF, São Paulo

- PAIGE R.M. (ed.) (1993a), Education for the intercultural experience, Intercultural Press, Yarmouth, Maine.

- PAIGE R.M. (1993b), Trainer competencies for international and intercultural programs, in R.M. PAIGE (ed.), Education for the intercultural experience (169-199), Intercultural Press, Yarmouth, Maine.

- PAIGE R.M. (2004), Instrumentation in intercultural training, in D. LANDIS, J.M. BENETT, M.J. BENNETT (eds.), Handbook of intercultural training. Third Edition (85-128), Sage, Thousand Oaks, CA.

- PASQUALOTTO G. (2007), Figure di pensiero. Opere e simboli nelle culture d'Oriente, Marsilio, Venezia.

- PIERIK R. (2004), Conceptualizing cultural groups and cultural difference. The social mechanism approach, in "Ethnicities", 4(4), 523-544.

- QUASSOLI F. (2006), Riconoscersi. Differenze culturali e pratiche comunicative, Raffaello Cortina Editore, Milano.

- RICOEUR P. (1965), De l'interprétation. Essai sur Freud, Seuil, Paris

- SAHLINS M. (1985), Islands of history, University of Chicago Press, Chicago,

- SAMOVAR L.A., PORTER R.E. (eds.) (1985), Intercultural communication. A reader. Fourth Edition, Wadsworth, Belmont, CA.

- SANDOVAL F.E.A. (2011) Educación intercultural indígena para la paz, articulo para la Conferencia ALAS 2011, Recife

- SANTOS DE SOUSA B. (2001), Toward an epistemology of blindness. Why the new forms of 'cerimonial adequacy' neither regulate nor emancipate, in "European Journal of Social Theory", 4(3), 251-279.

- SEVERINO E. (2007), L'identità della follia. Lezioni veneziane, Rizzoli, Milano.

- SHAFFER M.A., HARRISON D.A., GREGERSEN H., BLACK J.S., FERZANDI L.A. (2006), You can take it with you: Individual differences and expatriate effectiveness, in "Journal of Applied psychology", 91, 109-125.

- SPITZBERG B.H., CUPACH, W.R. (1984). Interpersonal communication competence, Sage, Londres.

- TING-TOOMEY S. (2004), Translating conflict face-negotiation theory into practice, in D. LANDIS, J.M. BENNETT, M.J. BENNETT (eds.), Handbook of intercultural training. Third Edition (217-248), Sage, Thousand Oaks, CA.

- VALSINER J. (2000), Culture and human development, Sage, Londra.

- VAN ASPEREN E. (2003), Interculturele communicatie \& ideologie, Pharos, Utrecht.

- VAN DYNE L., ANG S. (2005), Cultural intelligence: An essential capability for individuals in contemporary organizations, in http://global EDGE.msu. edu.

- VITERITTI A. (2005), Identità e competenze. Soggettività e professionalità nella vita sociale contemporanea, Guerini e Associati, Milano.

- YINGER R.J., HENDRICKS-LEE M.S. (1999), II pensiero pratico e la conoscenza al lavoro degli insegnanti, en F. ZAMBELLI, G. CHERUBINI, Manuale della scuola dell'obbligo: L'insegnante e i suoi contesti, Franco Angeli, Milano, 67-91.

- YUM J. O. (1994), The impact of Confucianism on interpersonal relationships and communication patterns in East Asia, in L.A. SAMOVAR, R.E. PORTER (eds.), Intercultural communication: A reader, Wadsworth, Belmont CA, 7586.

- ZHANG L, STERNBERG R.J. (2001), Thinking styles across cultures: Their relationships with student learning, en R.J. STERNBERG, L. ZANG (2001), Perspectives on thinking, learning, and cognitive styles (197-226), Lawrence Erlbaum, Londres

- ZHONG M. (1998), Perceived intercultural communication competence in cross-cultural interactions between Chinese and Americans, in "Critical Studies", 12, 161-179 


\section{Alessio Surian}

Alessio Surian desarrolla su acción docente y de investigador en el Departamento de Filosofia, Sociologia, Pedagogia y Psicologia Aplicada (FISPPA) la Universidad de Padua (Italia). Es formador y consultor en educación y políticas participativas y experto del programa Intercultural Cities. Trabaja con el Laboratorio de Políticas Públicas y con la Universidad Popular Urbana de la Alianza Internacional de Habitantes (www.habitants.org) donde es coordenador del Programa Memoria de Habitantes.

\section{LOS ASPECTOS \\ NO VERBALES EN \\ LA COMUNICACIÓN \\ INTERCULTURAL}

Ruth Vilà Baños

ruth_vila@ub.edu

RESUMEN

La comunicación intercultural es aquella comunicación de forma interpersonal donde intervienen personas con unos referentes culturales lo suficientemente diferentes como para que ello pueda suponer una barrera importante que altere la eficacia comunicativa. Se propone un análisis de la comunicación no verbal poniendo de manifiesto la importancia de los aspectos no verbales en la comunicación, así como las diferentes aportaciones de los estudios realizados sobre la kinésica, la prosémica, y algunos sentidos como el tacto y el olfato, altamente influidos por los referentes culturales propios de cada persona. Estos estudios ponen de manifiesto algunas diferencias básicas potenciales en un encuentro comunicativo intercultural.

Palabras claves: comunicación no verbal, interculturalidad, kinesia, prosémica.

\section{SUMMARY}

Intercultural communication is interpersonal communication involving people with cultural references different enough that it may pose a significant barrier that difficult communication efficiency. It proposes an analysis of nonverbal communication highlighting the importance of the nonverbal aspects of communication and the different contributions of studies on the kinesics, the proxemics and some senses like touch and smell, highly influenced by the cultural references of each person. These studies reveal some basic differences in potential intercultural communicative encounter. 